\title{
Management of neuroendocrine liver metastasis: Searching for new prognostic factor and appraising repeat hepatectomy
}

\author{
Junichi Arita, Sho Kiritani, Kiyoshi Hasegawa \\ Hepato-Biliary and Pancreatic Surgery Division, Department of Surgery, Graduate School of Medicine, University of Tokyo, Tokyo, Japan \\ Correspondence to: Kiyoshi Hasegawa. Professor and Chair, Hepato-Biliary and Pancreatic Surgery Division, Department of Surgery, Graduate School \\ of Medicine, University of Tokyo, 7-3-1 Hongo, Bunkyo-ku, Tokyo, Japan. Email: kihase-tky@umin.ac.jp. \\ Comment on: Armstrong EA, Beal EW, Shah M, et al. Radiographic characteristics of neuroendocrine liver metastases do not predict clinical outcomes \\ following liver resection. Hepatobiliary Surg Nutr 2020;9:1-12.
}

Submitted Feb 26, 2021. Accepted for publication Mar 17, 2021.

doi: 10.21037/hbsn-21-88

View this article at: http://dx.doi.org/10.21037/hbsn-21-88

Gastro-enteric and pancreatic neuroendocrine neoplasm (NEN) is categorized as a borderline malignant tumor and was historically called carcinoid, meaning carcinomamimicking. NEN tumors usually grow slower than most carcinoma tumors originating from visceral organs; however, some metastasize to lymph nodes or the liver, and are potentially life-threatening. One of the unique characteristics of NEN among various malignant or borderline malignant neoplasms is that tumor grade determined by histological examination has the strongest impact on patient prognosis. Differentiated NENs are classified as grade 1 to 3 on the basis of Ki- 67 value, and this grade correlates well with the development of lymph node and liver metastases, and even the overall survival (OS) of patients. However, this tumor grading is impossible unless a sufficient amount of tumor specimen is obtained by needle biopsy or tumor resection. Therefore, it would be useful if imaging features of NEN tumors could be used to predict tumor behavior, particularly when an indication of surgical resection is considered.

Armstrong et al. recently addressed the prognostic impact of enhancing patterns in dynamic computed tomography (CT) scans and calcification findings in plain CT scans in patients undergoing surgery for neuroendocrine liver metastasis (NELM) (1). In a previous study, enhancing patterns in primary NEN tumors identified on dynamic CT were analyzed, showing that a hypo-enhancing finding in the arterial phase was associated with higher tumor grade, lymph node metastasis, synchronous liver metastasis, and poorer OS (2). Another study demonstrated that a calcification finding in unenhanced $\mathrm{CT}$ of pancreatic NEN tumor was associated with higher tumor grade, larger tumor size, lymph node metastasis, and synchronous liver metastasis, although it did not impact on OS (3). Contrary to the results of these two previous studies, Armstrong et al. could not demonstrate any relationship with clinicopathological factors of either hypo-enhancing or calcification findings of NELM (1). They estimated that tumor biological behavior was worse or had already turned worse in patients with NELM, which may explain the above-mentioned discrepancies in the results. Because of the limited number of cases and the retrospective nature of their study, the results were not conclusive; however, their study is important in that prognostic factors obtained preoperatively were assessed in the field of NELM, for which the literature is scarce.

To analyze the long-term survival of patients undergoing surgical resection for NELM, the treatment choice for recurrent tumors after initial hepatic resection is important. However, no information regarding treatments for recurrent tumors was described in the study by Armstrong et al. Treatment choice after NELM recurrence was reported to be correlated with long-term survival of patients (4). Therefore, such information would help the understanding of the above-mentioned discrepancies; in particular, the number of patients undergoing surgical resection of recurrent tumors in each imaging category would be useful. If enhancing patterns or calcified findings of NELM tumors in CT are correlated with tumor biology, the situations of NELM tumors at recurrence, 
namely, tumor number, tumor size, or distribution in the liver, which may impact on the resectability of recurrent tumors, would differ according to such imaging findings. From another point of view, differences in the proportion of patients undergoing resection of recurrent tumors may affect the long-term results. Some patients in whom resection is possible but abandoned on the basis of pessimistic idea for tumor recurrence may lose the chance of long-term survival.

The majority of patients undergoing initial NELM resection experience tumor recurrence, most of which is observed in the liver. However, a consensus of the treatment strategy for recurrent tumors has not yet been achieved, mainly because of the paucity of the literature. Recently, we reported the efficiency of repeat hepatectomy of recurrent NELM tumors through an investigation of the long-term results of patients with recurrence (5). This study reported a higher 5-year OS rate (79\%) after repeat hepatectomy than that without $(35 \%)$. Furthermore, the recurrencefree survival (RFS) curve after initial hepatectomy was similar to that after repeat hepatectomy, which also indicated the usefulness of repeat hepatectomy. Another study by Spolverato et al. demonstrated the utility of repeat hepatectomy for recurrent NELMs through a multiinstitutional retrospective study (6). In this study, repeat hepatectomy of recurrent tumors resulted in a 10 -year OS rate of $60 \%$.

Another concern in the field of NELM treatment is whether a 'cure' can be achieved using an aggressive strategy. Although OS after resection of NELM is not desperate because of the slow-growing nature of NEN tumor, most patients develop recurrence after initial hepatectomy of NELM. Through strict postoperative follow-up of the patients at our own outpatient clinic, 10-year RFS after initial hepatectomy was less than 10\% (5). In the field of surgery for colorectal liver metastasis, RFS after initial hepatectomy is also low; however, a cure can be expected after one or more repeat hepatectomies even after tumor recurrence (7). In this context, we recently calculated the recurrence-free interval after the 'last' hepatectomy, in other words, the 'most recent' hepatectomy for NELM in each patient. Among 49 patients undergoing initial hepatectomy for NELM, the longest 'last' recurrence-free interval was 14.8 years, followed by 9.8 years, and 7.3 years (data not published). Disease cure after NELM resection is possible, though not expected in many patients. Moreover, a strategy using aggressive repeat hepatectomies provides favorable long-term survival [10-year OS rates of $52 \%$ (6) and $70 \%(5)]$.

To improve the prognosis of patients with NELM, the timing and indication of surgical resection of initial and recurrent NELM tumors are critical because surgical resection has the strongest prognostic impact, but a high rate of tumor recurrence is estimated. Although Armstrong et al. could not identify any CT findings as prognostic factors (1), it would be useful to determine the surgical indication and timing based on any factor that is available before surgery; for example, imaging findings of not only CT scans, but also magnetic resonance imaging, fluorodeoxyglucose positron emission tomography, and somatostatin receptor scintigraphy that predict the efficiency of surgery.

\section{Acknowledgments}

We thank H. Nikki March, PhD, Edanz Group (https:// en-author-services.edanz.com/ac) for editing a draft of this manuscript.

Funding: This work was supported by JSPS KAKENHI Grant Number 19K09138.

\section{Footnote}

Provenance and Peer Review: This article was commissioned by the editorial office of Hepatobiliary Surgery and Nutrition. The article did not undergo external peer review.

Conflicts of Interest: All authors have completed the ICMJE uniform disclosure form (available at https://hbsn.amegroups. com/article/view/10.21037/hbsn-21-88/coif). Dr. KH serves as an unpaid editorial board member of Hepatobiliary Surgery and Nutrition. The other authors have no conflicts of interest to declare.

Ethical Statement: The authors are accountable for all aspects of the work in ensuring that questions related to the accuracy or integrity of any part of the work are appropriately investigated and resolved.

Open Access Statement: This is an Open Access article distributed in accordance with the Creative Commons Attribution-NonCommercial-NoDerivs 4.0 International License (CC BY-NC-ND 4.0), which permits the noncommercial replication and distribution of the article with the strict proviso that no changes or edits are made and the original work is properly cited (including links to both the 
formal publication through the relevant DOI and the license). See: https://creativecommons.org/licenses/by-nc-nd/4.0/.

\section{References}

1. Armstrong EA, Beal EW, Shah M, et al. Radiographic characteristics of neuroendocrine liver metastases do not predict clinical outcomes following liver resection. Hepatobiliary Surg Nutr 2020;9:1-12.

2. Worhunsky DJ, Krampitz GW, Poullos PD, et al. Pancreatic neuroendocrine tumours: hypoenhancement on arterial phase computed tomography predicts biological aggressiveness. HPB (Oxford) 2014;16:304-11.

3. Poultsides GA, Huang LC, Chen Y, et al. Pancreatic neuroendocrine tumors: radiographic calcifications correlate with grade and metastasis. Ann Surg Oncol 2012;19:2295-303.

Cite this article as: Arita J, Kiritani S, Hasegawa K. Management of neuroendocrine liver metastasis: Searching for new prognostic factor and appraising repeat hepatectomy. HepatoBiliary Surg Nutr 2021;10(3):410-412. doi: 10.21037/ hbsn-21-88
4. Saxena A, Chua TC, Zhao J, et al. Liver-directed therapy for neuroendocrine neoplasm hepatic metastasis prolongs survival following progression after initial surgery. J Surg Oncol 2012;105:342-50.

5. Kiritani S, Arita J, Matsumura M, et al. Repeat hepatectomy for patients with recurrent neuroendocrine liver metastasis: Comparison with first hepatectomy. Surgery 2020;167:404-9.

6. Spolverato G, Bagante F, Aldrighetti L, et al. Management and outcomes of patients with recurrent neuroendocrine liver metastasis after curative surgery: An international multi-institutional analysis. J Surg Oncol 2017;116:298-306.

7. Oba M, Hasegawa K, Shindoh J, et al. Survival benefit of repeat resection of successive recurrences after the initial hepatic resection for colorectal liver metastases. Surgery 2016;159:632-40. 